\title{
Theory of Coil Antennas
}

\author{
Trilochan Padhi \\ Division of Engineering and Applied Physics, Harvard University, Cambridge, Mass.
}

(Received October 21, 1964; revised December 12, 1964)

\begin{abstract}
In this paper, a method is presented by which the distribution of current on some structurally simple coil or multiturn loop antennas may be obtained. The input admittances of unshielded and shielded coils are determined and their operation as receiving elements is considered.
\end{abstract}

\section{Introduction}

The high-frequency behavior of thin circular loop antennas has been studied in the past by several investigators [Wu, 1962]. It is shown in this paper how results previously obtained for single-turn loop antennas can be applied to give the admittance and distribution of current for coils that possess symmetry in the sense that all turns of the coil are equivalent. Such symmetry obtains if at any section of the coil the wires appear at the corners of a regular polygon, and further if the main diagonal of this polygon is much smaller than both the radius of the coil and the wavelength in air.

One advantage of the coil over the single-turn loop antenna is that at low frequencies, the output voltage is directly proportional to the number of turns of the coil when it is used as a receiving antenna. Also its higher impedance may make tuning or matching a simpler problem. For these reasons coils are widely used in direction finders and radio receivers. A recent application of coils is in the production of very high rf magnetic fields. The present theory may be used to calculate the voltage distribution on the coil and hence estimate the maximum magnetic field obtainable before electrical breakdown occurs.

In practice, the detailed structure of the coil may not permit rigorous application of the following analysis but in most cases it should be possible to obtain semiquantitative estimates.

In brief, the method is as follows. Instead of solving directly for the current on each of the $N$ turns of the coil, it is found more convenient to regard the current on each turn as the superposition of currents in $N$ phase sequences. In the sequence for which the phase difference between the currents on any two turns is zero, the current distribution is the same as that on a single-turn loop of appropriate equivalent cross-sectional size. Other sequences are nonradiating and behave essentially like the TEM mode on a two-wire line. The current in each sequence is, therefore, known or can be found, and the current and input admittance of the coil determined by summing up the contributions from each phase sequence.

\section{Current Sequences}

Let $N$ be the number of turns on the coil. A point on the coil is denoted by $\chi$ which is its angular distance from some fixed point on the coil, measured along the conductor. The current $I(\chi)$ across the section of the conductor at $\chi$ must satisfy the periodicity condition

$$
I(\chi)=I(\chi+2 \pi N) \text {. }
$$

Hence, $I(\chi)$ may be written in a Fourier series:

$$
I(\chi)=\sum_{m=-\infty}^{\infty} A_{m} e^{-j m \chi / N}
$$

To display this as a sum of phase sequences put

$$
\begin{aligned}
& m=k+n N ; \quad(k=0,1,2, \ldots ., N-1 ; \\
& n=0, \pm 1, \pm 2, . . . \pm \infty) .
\end{aligned}
$$

An equivalent form for $I(\chi)$ is, therefore,

$$
I(\chi)=\sum_{k=0}^{N-1} e^{-j k \chi / N} \sum_{n=-\infty}^{\infty} A_{n}^{k} e^{-j n \chi}
$$

The $(1+i)$ th turn of the coil is defined by

$$
\chi=2 \pi i+\psi ;(-\pi<\psi \leqslant \pi) .
$$

Hence the current on the $(1+i)$ th turn is

$$
\begin{aligned}
I_{i}(\psi) & =\sum_{k=0}^{N-1} e^{-2 \pi i j k / N}\left\{e^{-j k \psi / N} \sum_{n=-\infty}^{\infty} A_{n}^{k} e^{-j n \psi}\right\} \\
& =\sum_{k=0}^{N-1} e^{-2 \pi i j k / N} I^{k}(\psi),
\end{aligned}
$$

where

$$
I^{k}(\psi)=e^{-j k \psi / N} \sum_{n=-\infty}^{\infty} A_{n}^{k} e^{-j n \psi} .
$$

$I^{k}(\psi)$ is called the $k$-sequence current. When a pure 
$k$-sequence current exists on the coil, it is implied that the current on the $(1+i)$ th turn is

$$
I_{i}^{k}(\psi)=e^{-2 \pi i j k / N} I^{k}(\psi) .
$$

It follows from (2) that

$$
I_{\text {tot }}^{k}(\psi) \equiv \sum_{i=0}^{N-1} I_{i}^{k}(\psi)=N I^{k}(\psi) \delta_{k 0}
$$

that is, in each sequence $k \neq 0$, the total current across a section of the coil is zero.

\section{Driving Voltage Sequences}

Suppose a voltage generator of strength $V_{i}$ is introduced into each of the $N$ turns at $\psi=0$ (fig. 2). Define $N$ sequence voltages $V^{k}$ by the set of equations

$$
V_{i}=\sum_{k=0}^{N-1} e^{-2 \pi i j k / N} V^{k} ;(i=0,1, \ldots, N-1) .
$$

The solution of (4) for $V^{k}$ is

$$
V^{k}=\frac{1}{N} \sum_{i=0}^{N-1} e^{2 \pi i j k / N} V_{i}
$$

For the case where only one source is present (say in the first turn), this simplifies to

$$
V^{k}=V \mid N, \text { where } V_{i}=V \delta_{i 0} .
$$

\section{Phase Sequence Input Admittances}

Great simplification of the problem of coil antennas results if it is assumed that there is sufficient symmetry to ensure that if the coil is driven by a $k$-sequence voltage, the currents, too, form the same sequence. Coils that approximately satisfy this condition may be wound by making sure that at any section, the turns of the coil appear at the corners of a regular polygon. Further, since all turns should be as nearly as possible of the same length (physically as well as electrically), the separation between turns should be small compared to both the radius of the coil and the wavelength corresponding to the frequency of operation. A fourturn coil is shown in figure 1 ; for clarity the separation between turns has been exaggerated.

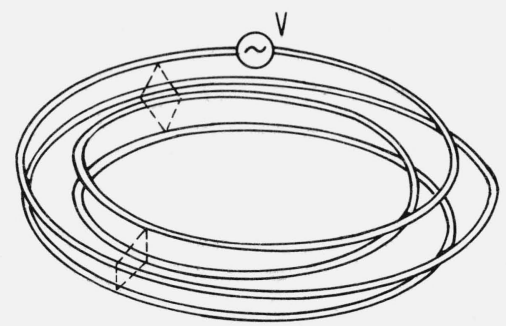

Figure 1. A four turn coil in which the conductors appear at the corners of a square in every cross section.
Consider first the distribution of current induced by a zero-sequence voltage. By suitably altering the connections at the $N$ driving points, the problem of the coil reduces to that of $N$ identical, closely coupled, circular antennas driven symmetrically, i.e., by equal voltages in phase. The change in connections leaves the distribution of current unaffected. It is shown in the literature [King and Harrison, 1965] that for two identical, closely coupled loop antennas driven in zero-phase sequence, the distribution of current is the same as that on an isolated loop of the same diameter, but with the wire of radius $a$ replaced by wire of radius $\bar{a}=\sqrt{(a d)}$, where $d$ is the separation. A simple extension of this result to the present case shows that the equivalent loop is one with the same mean diameter as the coil but made of wire of radius $\bar{a}$ given by

$$
\bar{a}=\sqrt[N]{a d_{1} d_{2} \ldots d_{N-1}}
$$

where $d_{i}$ is the distance of the $(1+i)$ th turn from the first turn. The current $I^{\mathrm{loop}}(\psi)$ and admittance $y^{\text {loop }}$ for the equivalent loop have been evaluated for small $\bar{a}$ in an earlier reference [Wu, 1962], and numerical results are available [King, Harrison, and Tingley, 1963]. In terms of these known quantities the zerosequence current on the coil is, therefore,

$$
I^{0}(\psi)=\frac{1}{N} I^{\mathrm{loop}}(\psi)
$$

The zero-sequence input admittance is defined by

$$
y^{0} \equiv \frac{I^{0}(0)}{V^{0}}=\frac{1}{N} \frac{I^{\text {loop }}(0)}{V^{0}} .
$$

Hence

$$
y^{0}=\frac{1}{N} y^{\text {loop }}
$$

For all higher sequences, radiation from the coil can be neglected as a consequence of (3) and the condition that the separation between any pair of turns be much smaller than a wavelength. Now the theory of a straight, infinitely long, multiwire transmission-line

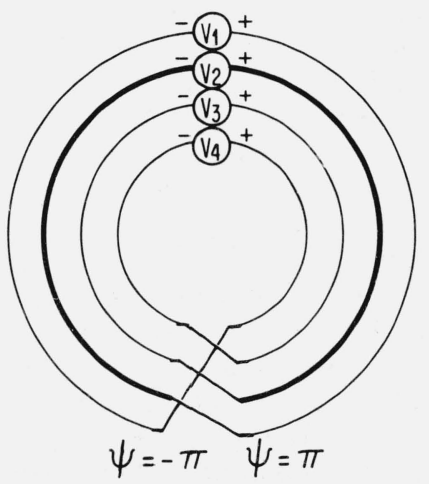

FIGURE 2. Schematic view of coil. One turn is drawn with a heavy line. 
[Tai, 1948] shows that when it is driven by a $k$ sequence voltage $(k \neq 0)$, a TEM wave propagates along the line with the velocity of light. Propagation along the line is essentially unaffected even if the line is curved, provided the radius of curvature is much larger than the separation between conductors. Hence, the $k$-sequence current $I^{k}(\psi)$ and (in analogous notation) the $k$-sequence scalar potential $\phi^{k}(\psi)$ obey (for $\psi \neq 0$ ) the equations

$$
\frac{1}{R^{2}} \frac{d^{2} \phi^{k}}{d \psi^{2}}+\beta^{2} \phi^{k}=0
$$

and

$$
I^{k}=\frac{j}{\beta R Z^{k}} \frac{d \phi^{k}}{d \psi}
$$

where

$R=$ mean radius of the coil,

$Z^{k}=$ characteristic impedance, i.e., impedance required in each arm of a star-connected load that matches the transmission-line when it is excited by a $k$-sequence voltage,

and

$\beta=$ phase constant of the em wave.

At $\psi=0$ there is a discontinuity in scalar potential $($ see $(5 a))$

$$
\phi^{k}(0+)-\phi^{k}(0-) \equiv V^{k}=V / N .
$$

Further, (1) gives

$$
I^{k}(-\pi)=e^{2 \pi j k / N} I^{k}(\pi)
$$

and, analogously

$$
\phi^{k}(-\pi)=e^{2 \pi j k / N} \phi^{k}(\pi)
$$

The solution of (7) and (8) subject to (9), (10), and (11) is obtained in the appendix. The $k$-sequence input admittance is shown to be

$$
\begin{array}{r}
y^{k} \equiv \frac{I^{k}(0)}{V^{k}}=\frac{j}{4 Z^{k}} \frac{\sin 2 \pi \beta R}{\sin \pi\left(\frac{k}{N}+\beta R\right) \sin \pi\left(\frac{k}{N}-\beta R\right)} ; \\
(k \neq 0) .
\end{array}
$$

Equations (6) and (12) give the input admittance for every sequence, $k=0,1,2, \ldots ., N-1$.

It is now a simple matter to calculate the admittance $y_{\text {in }}$ presented to the source at $\psi=0$ on the first turn:

$$
y_{\text {in }}=I_{0}(0) / V=\sum_{k=0}^{N-1} y^{k} / N
$$

Hence

$$
\begin{aligned}
y_{\text {in }}=\frac{y^{\text {loop }}}{N^{2}} & +j \frac{\sin 2 \pi \beta R}{4 N} \\
& \times \sum_{k=1}^{N-1} \frac{1}{Z^{k} \sin \pi\left(\frac{k}{N}+\beta R\right) \sin \pi\left(\frac{k}{N}-\beta R\right)} .
\end{aligned}
$$

It is interesting to note that for $N \beta R<<1$, (13) reduces to the familiar low-frequency formula, $y_{\text {in }}=y^{\text {loop }} / N^{2}$. In fact, as long as the coil is perfectly conducting and the medium surrounding it is lossless, the input conductance is always $1 / N^{2}$ times the conductance of the equivalent loop, irrespective of the size of the coil.

Another well-known feature that follows from (13) is the antiresonance associated with the distributed capacitance between the several pairs of turns. At low frequencies, the input admittances of all sequences other than the zero-sequence are capacitive, while the zero-sequence input admittance is inductive. The net susceptance becomes zero at a frequency that is quite low. For some typical cases calculated below, antiresonance occurs at $N \beta R \sim 0.2$; i.e., the total length of wire used in the coil is only a fifth of a wavelength.

Calculated values of susceptance for coils of 2,3 , and 6 turns are shown in figure 3. For each coil the distance between adjacent turns has been taken as twice the diameter of the wire used. The actual size of the wire is chosen such that $2 \ln 2 \pi R / \bar{a}=10$. In order to show the resonances clearly, the scale for the ordinate in figure 3 is chosen to be proportional to the arc tangent of the susceptance (multiplied by a constant). It will be noticed that the behavior of the input susceptance resembles that of a short-circuited transmission line quite closely except where $\beta R$ is near an integer. At points of antiresonance or zero susceptance, the radiation from the coil is significant. However, where the susceptance becomes infinite, one of the transmission-line modes effectively short circuits all others and radiation is a minimum. This is the condition of resonance. Resonance occurs whenever $\beta R=n+k / N ; \quad(k=1,2, \ldots, N-1$; $n=0,1,2, \ldots, \infty)$.

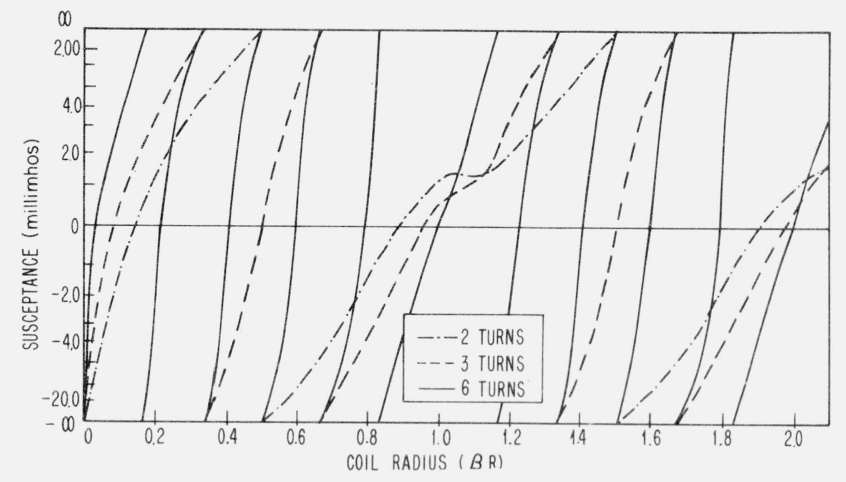

FIGURE 3. Susceptance of coil antennas. 


\section{Shielded Coil Antenna}

A method almost identical to that employed above may be used to analyze the shielded coil or multiturn loop antenna. In its most common form, a shielded coil antenna consists of a coil threaded through a conducting tube that is bent into the form of a ring. The ends of the tube are brought close together but do not meet. Here too, the method only applies to coils of the kind described in earlier sections, enclosed in shields that do not disturb their symmetry. An idealized configuration is considered, in which the generator (or load) in series with the coil is itself within the shield. For convenience, the gap in the shield is taken at $\psi=\pi$, (where $\psi=0$ is the position of the generator). It is assumed throughout that the shield is thin and perfectly conducting, and that the diameter of its cross section is much smaller than a wavelength.

The current on the coil and, similarly, the scalar potential, may be decomposed into sequences exactly as in section 2 . For any $k$-sequence, $k \neq 0,(7),(8)$, (9), (10), and (11) hold because, as a consequence of (3), the gap in the shield at $\psi=\pi$ does not affect its behavior. Hence, for $k \neq 0$, the $k$-sequence input admittance is given by (12). Of course, the characteristic impedance $Z^{k}$ will now be different because of the presence of the shield.

The current $I^{\text {sh }}(\psi)$ across a section of the shield at $\psi$ may be split into two parts:

$$
I^{\mathrm{sh}}(\psi)=I^{\mathrm{rad}}(\psi)-N I^{0}(\psi)
$$

Let $\phi^{\text {sh }}(\psi)$ be the scalar potential on the shield. The zero-sequence potential difference between the coil and the shield is

$$
\phi^{\text {diff }}(\psi)=\phi^{0}(\psi)-\phi^{\text {sh }}(\psi) .
$$

$I^{0}(\psi)$ and $\phi^{\text {diff }}(\psi)$ together form a nonradiating, transmission-line type of wave, that exists within the shield. On the other hand, the part $I^{\mathrm{rad}}(\psi)$ of the current on the shield radiates like the current on a loop of the same shape and size as the shield. Hence, in terms of the admittance of this equivalent loop,

$$
I^{\mathrm{rad}}(\pi)=y^{\mathrm{loop}}\left[\phi^{\mathrm{sh}}(-\pi)-\phi^{\mathrm{sh}}(\pi)\right] .
$$

The loop mode and the transmission-line mode couple through the gap at $\psi=\pi$ where the following equations must hold:

and

$$
I^{\operatorname{rad}}(\pi)=N I^{0}(\pi)
$$

$$
\phi^{\mathrm{diff}}(\pi)-\phi^{\mathrm{diff}}(-\pi)=\phi^{\mathrm{sh}}(-\pi)-\phi^{\mathrm{sh}}(\pi) .
$$

Hence

$$
\phi^{\text {diff }}(\pi)-\phi^{\text {diff }}(-\pi)=I^{0}(\pi) \times N=y^{\text {loop }} .
$$

Thus, in effect, the loop mode places an impedance $N / y^{\text {loop }}$ in series with each turn of the coil at $\psi=\pi$.
The zero-sequence input admittance is now readily found to be

$$
y^{0}=\frac{1}{2 Z^{0}} \frac{2 Z^{0} y^{\text {loop }}+j N \tan \pi \beta R}{N+2 Z^{0} y^{\text {loop }} \tan \pi \beta R} .
$$

Using (12) and (14), the input admittance is obtained from

$$
y^{\mathrm{in}}=\sum_{k=0}^{N-1} y^{k} / N .
$$

Formulas for $Z^{k}$ to be used in calculating $y^{k}$ are given in the appendix.

\section{Receiving Coil Antenna}

The unshielded coil is considered first. Assume that the effective length [King and Harrison, 1965] of the equivalent loop is known and that the coil is loaded by an impedance $R$ at $\psi=0$ on the first turn. From the definition of effective length it follows that if all turns of the coil were open-circuited at $\psi=0$, the resulting potential difference across the opencircuit would be the same for all turns and would be given by

$V^{\text {ind }}=-2 \mathbf{h}_{e} \cdot \mathbf{E}^{\text {inc }}$

where

$\mathbf{E}^{\text {inc }}=$ incident electric field

and

$2 \mathbf{h}_{e}=$ effective height of the equivalent loop; $\mathbf{h}_{e}$ is a function of the direction of propagation of the incident field and its polarization.

In normal operation as a receiving element, the voltage sequences must be given by

$$
V^{k}=-R I_{0}(0) / N=-R \sum_{k=0}^{N-1} I^{k}(0) / N .
$$

But, in terms of the current sequences $I^{k}(0)$,

$$
V^{k}=V^{\text {ind }} \delta_{k 0}+I^{k}(0) / y^{k} .
$$

Using this and (16) to solve for $\Sigma I^{k}(0)$ yields

$$
\sum_{k=0}^{N-1} I^{k}(0)=-\frac{1}{R+1 / y^{\text {in }}}\left(\frac{y^{0}}{y^{\text {in }}} V^{\text {ind }}\right) .
$$

The Thevenin equivalent of the coil at its output terminals is, therefore, a voltage generator of strength $-y^{0} V^{\text {inc }} / y^{\text {in }}$ in series with an impedance $1 / y^{\text {in }}$. Alternatively, this may be regarded as a current generator of strength $-y^{0} V^{\text {ind }}$ in parallel with an admittance, $y^{\text {in }}$.

The derivation from this simple picture, of the lowfrequency result that the output voltage of a coil is proportional to the number of turns, is interesting. First it will be noted that since $y^{0}=y^{\text {loop}} / N$, the strength 
of the current generator decreases as $N$ is increased and is in fact roughly proportional to $1 / N$. But at low frequencies, $y^{\text {in }}$ is proportional to $1 / N^{2}$. Hence, at low frequencies, the open-circuit output voltage is proportional to the number of turns.

The equivalent circuit of a shielded receiving coil may be obtained by a similar method. Here, a simpler method will be used, which rests on the fact that the internal admittance of the shielded loop, viewed from its terminals, is the same whether it is being used as a transmitter or a receiver. The strength of the current generator in parallel with the internal admittance is found by calculating the current that flows through the output terminals when they are short circuited. In this condition, only zero-sequence currents are excited. Using elementary transmission-line theory, it can be shown that the strength of the equivalent current generator, when viewed from the terminals at $\psi=0$ on the first turn, is

$$
=-\frac{V^{\text {ind }} y^{\text {loop }}}{N \cos \pi \beta R+2 j y^{\text {loop }} Z^{0} \sin \pi \beta R} \text {. }
$$

Here $y^{\text {loop }}$ and $Z^{0}$ have the meaning they had in section 5 , and $V^{\text {ind }}$ is the voltage induced across the gap of the equivalent loop, open-circuited at $\psi=\pi$. Just as in the unshielded case (15), one may write down $V^{\text {ind }}$ in terms of an effective length, but care should be taken to see that it refers to a load situated at $\psi=\pi$.

\section{Appendix}

The solution of the wave equation (7), subject to the boundary conditions (9) and (11) is

$$
\begin{aligned}
\phi^{k}(\psi) & =\frac{1}{2} V^{k} \frac{|\psi|}{\psi} \frac{\sin \beta R(\pi-|\psi|)}{\sin \beta R \pi} \\
& +\frac{\phi^{k}(\pi)}{\sin 2 \beta R \pi}\left[\sin \beta R(\pi+\psi)+e^{2 \pi j k / N} \sin \beta R(\pi-\psi)\right] .
\end{aligned}
$$

Equation (8) now gives

$$
\begin{aligned}
I^{k}(\psi) & =\frac{j}{Z^{k}}\left\{-\frac{V^{k}}{2} \frac{\cos \beta R(\pi-|\psi|)}{\sin \beta R \pi}\right. \\
& \left.+\frac{\phi^{k}(\pi)}{\sin 2 \beta R \pi}\left[\cos \beta R(\pi+\psi)-e^{2 \pi j k / N} \cos \beta R(\pi-\psi)\right]\right\} .
\end{aligned}
$$

This expression for $I^{k}(\psi)$ must satisfy the boundary condition (10). This is possible if and only if

$$
\phi^{k}(\pi)=-j V^{k} \frac{\cos \beta R \pi \sin \frac{k \pi}{N} e^{-j \pi k / N}}{\cos \frac{2 \pi k}{N}-\cos 2 \pi \beta R} .
$$

Substitution from (A2) for $\phi^{k}(\pi)$ in (A1) leads to

$$
\begin{array}{r}
y^{k}=\frac{I^{k}(0)}{V^{k}}=\frac{j}{4 Z^{k}} \frac{\sin 2 \pi \beta R}{\sin \pi\left(\frac{k}{N}+\beta R\right) \sin \pi\left(\frac{k}{N}-\beta R\right)} ; \\
(k \neq 0) .
\end{array}
$$

The only quantity that remains to be calculated is $Z^{k}$. The formula (neglecting proximity) to be used in calculating the sequence admittance for the unshielded coil is avialable [Tai, 1948] for $k=1$. The generalization to other values of $k$ is not difficult and yields

$$
Z^{k}=60\left[\ln \frac{d}{a}-2 \sum_{i=1}^{S} \cos \frac{2 \pi i k}{N} \ln \sin \frac{\pi i}{N}\right] \text { ohms }
$$

For the shielded coil, the formula to be used is (for $a<<d / N, D-d)$

$$
\begin{aligned}
& Z^{k}=60\left[\ln \frac{d}{a} \frac{\left(1-d^{2} / D^{2}\right)}{\left(1+d^{2} / D^{2}\right)}+N \delta_{k 0} \ln \frac{D}{2 d}\left(1+\frac{d^{2}}{D^{2}}\right)\right. \\
& \left.-2 \sum_{i=1}^{S} \cos \frac{2 \pi i k}{N} \ln \frac{1}{\Delta_{i}} \sin \frac{\pi i}{N}\right] \text { ohms. }
\end{aligned}
$$

In (A4) and (A5),

$d=$ diameter of the circle in which the regular polygon defined by the conductors may be inscribed, $a=$ radius of each conductor,

$S=N / 2$ or $(N-1) / 2$, whichever is an integer,

$D=$ diameter of a cross section of the shield, if any,

and

$$
\Delta_{i}=\sqrt{1+\frac{d^{4}}{D^{4}}-\frac{2 d^{2}}{D^{2}} \cos \frac{2 \pi i}{N}} /\left(1+\frac{d^{2}}{D^{2}}\right)
$$

In these formulas, the proximity effect is neglected. A study of the exact and approximate formulas for the case of the two-wire line shows that no serious error results if the approximation is made for $d / a \geqslant 4$. Hence, in dealing with the multiwire line, one can hope for reasonable accuracy provided care is taken to use the approximate formulas only when the side of the polygon is at least four times the radius of the conductor.

The author is grateful to R. W. P. King for his guidance and kind encouragement.

\section{References}

King, R.W.P., and C. W. Harrison, Jr. (1965), Electromagnetic Radiation, ch. IX, sec. 7, and ch. X, sec. 2 (to be published).

King, R. W. P., C. W. Harrison, Jr., and D. G. Tingley (1963), The admittance of the bare circular loop antennas in a dissipative medium, Sandia Corporation Monograph, SCR-674.

Tai, C. T. (1948), High-frequency polyphase transmission-lines, Proc. IRE 36, 1370.

Wu, T. T. (1962), Theory of the thin circular loop antenna, J. Math. Phys. 3, 1301-1304, and the references given therein. 